\section{Furcht, Angst, Angsterkrankungen}

\author{
Hans-Christian Pape
}

Aus biologischer Sicht sind Furcht (das Gefühl der konkreten Bedrohung beispielsweise durch eine Person oder ein Objekt) und Angst (das diffuse Unbehagen in einer als bedrohlich empfundenen Situation) wichtige Komponenten unseres Verhaltensrepertoires: Sie schützen uns vor potenziell unangenehmen oder sogar schädlichen Einflüssen. So lernen wir durch Sozialisierung oder schmerzvolle Erfahrung, bestimmte Verhaltensweisen oder Begegnungen zu vermeiden, aus Angst davor, verletzt zu werden. Charles Darwin hat bereits im Jahr 1872 darauf hingewiesen (C Darwin. The expression of the emotions in man and animals. John Murray, London, 1872), dass diese stammesgeschichtlich alten Merkmale (,horror and agony") in allen Säugetieren, wenn nicht sogar in allen Wirbeltieren vorhanden sind. Der phylogenetisch alte Ursprung dieser Verhaltensreaktionen und deren positive Selektion im Verlauf der Evolution sind leicht nachvollziehbar: Individuen, die in einer gefährlichen Umwelt furchtsam reagieren, überleben besser. So ist der bei uns als ,ängstlich“ diskriminierte Hase in der chinesischen Mythologie das Sinnbild der Langlebigkeit. Oder anders herum: „Die Mutigen sterben zuerst".

Allerdings können anhaltende Veränderungen oder eine extreme Störung dieser Verhaltensmechanismen $\mathrm{zu}$ exzessiven und in Bezug auf die Situation unangemessenen Reaktionen führen, die durch den Betroffenen kaum kontrollierbar sind: zum Beispiel eine andauernde Angst lange nach Ende der eigentlich bedrohlichen Situation, ein Wiedererleben von Erfahrungen mit extremer Angst, oder eine omnipräsente, für den Außenstehenden kaum erklärliche basale Ängstlichkeit. Panikstörung, Phobien, posttraumatische Belastungsstörung und generalisierte Angsterkrankung.

„Now is the age of anxiety "(W. H. Auden. The age of anxiety: a baroque eclogue. Random House, New York 1947). Obwohl die barocke Lyrik von Wystan Hugh Auden bereits vor mehr als 60 Jahren mit dem Pulitzer-Preis gewürdigt wurde, ist dessen Kernaussage zu Beginn des 21. Jahrhunderts aktueller denn je: Die Zahl Angsterkrankter ist besorgniserregend, mit einer Wahrscheinlichkeit der Erkrankung im Verlaufe des Lebens von 14-29\% (in der Europäischen Union) und einem Krankheitsbeginn zum Teil schon im Alter von 11 Jahren. Damit

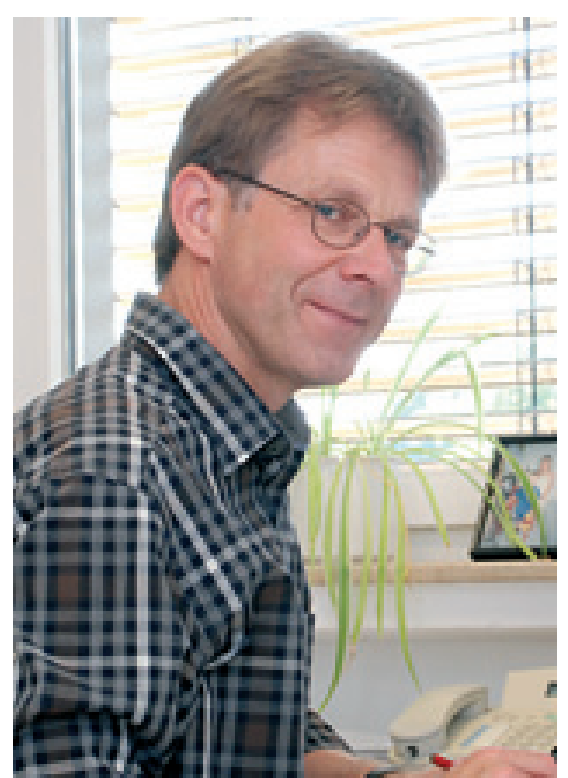

zählen Angsterkrankungen nicht nur zu den häufigsten psychischen Störungen, sondern auch zu denjenigen mit frühestem Krankheitsbeginn. Die Krankheit führt zu erheblichen Beeinträchtigungen von Lebensqualität, Lernvermögen, Arbeits- und Berufstätigkeit. Der Erkrankung liegt ein komplexes Wechselspiel aus genetischer Disposition, psycho-sozialen und autobiografischen Spezifika (Life History) in Verbindung mit neuropathologischen Veränderungen der „Furchtschaltkreise“ des Gehirns zugrunde. Allerdings bleiben Ätiologie und Pathogenese der Angsterkrankungen in großen Teilen unverstanden, was erhebliche Probleme in der diagnostischen Einordnung und insbesondere in der Entwicklung wirksamer Therapieverfahren mit sich bringt. In der Tat dokumentieren epidemiologische Studien die enorme ökonomische Relevanz der Angsterkrankungen und verweisen auf die aus den Erkrankungen resultierenden Herausforderungen für die Systeme der

Eine systematische, wissenschaftliche Analyse dieser Emotionen und ihrer pathologischen Alteration erfordert Ansätze der Neurobiologie, Genetik, Psychologie und Psychiatrie, in Verbindung mit einem translationalen Konzept. Dieses sollte einerseits auf die Erfassung von Detailmechanismen mit einer notwendigerweise reduktionistischen Grundanlage zielen; es sollte andererseits in hinreichender BreiGesundheitsfürsorge. te translationale Ansätze und konkrete Schlussfolgerungen in Richtung einer Diagnose- und Therapieentwicklung sowie der Erstellung von individuellen Risikoprofilen für die Erkrankungen zulassen. Mit diesem strategischen Ziel wurde der translationale Sonderforschungsbereich „Furcht, Angst, Angsterkrankungen (SFB-TRR58)“ im Juli 2008 von der Deutschen Forschungsgemeinschaft eingerichtet und im Jahre 2012 in die aktuell 2. Förderperiode verlängert (http://sfbtrr58.uni-muenster.de/). Dieser Sonderforschungsbereich vereint Wissenschaftlerinnen und Wissenschaftler der Universitäten und Universitätsklinika aus Münster, Hamburg und Würzburg sowie eine eng kooperierende Gruppe der Universität Mainz. Die Mitglieder bringen ihre Expertise aus Molekularbiologie, Genetik, Neurophysiologie, Verhaltensbiologie, Psychologie, Psychiatrie und der Bildgebung ein. Diese einzigartige Bündelung von Expertise und interdisziplinärer Zusammenarbeit in diesem Forschungsgebiet schafft die Grundlage für eine wissenschaftliche Strategie, in der sich experimentelle Konzepte in tierexperimentellen Studien und Untersuchungen am Menschen gezielt ergänzen. Damit ist die Voraussetzung gegeben, molekulare und systemische Mechanismen von Furcht, Angst und Angsterkrankungen nicht nur inkrementell in Details zu charakterisieren, sondern grundlegende Prinzipien dieser Zustände zu entschlüsseln. Aktuell besteht der SFB-TRR58 aus 19 Teilprojekten, die in drei Bereichen und einem Zentralbereich organisiert sind: (A) Tierexperimentelle Studien, (B) Präklinische Studien, (C) Klinische Translation und Intervention, (Z02) Funktionelle Genomik und Gen $x$ UmweltWechselwirkung.

Die wissenschaftliche Strategie zur Durchführung und Weiterentwicklung des Forschungsprogramms des SFB-TRR58 folgt zwei Hauptlinien. Entlang einer Linie fokussieren wir auf ausgewählte Modelle, Mechanismen und Erkrankungen, generieren konvergente Ansätze aus verschiedenen Beschreibungsebenen und gewährleisten damit ein Maximum an Synergieeffekten. Dieser Ansatz ist bewusst reduktionistisch ausgelegt. Entlang einer zweiten strategischen Linie haben wir screening Ansätze implementiert, die auf Kandidatengene und „,innovative“ Gene für Angsterkrankungen zielen. Nach Identifizierung eines Gens wird dessen Relevanz in einer Art ,proofof-principle" mithilfe der etablierten experimentellen Modelle und Konzepte überprüft, wobei die zuvor erreichte Kenntnis der Detailfunktionen eine entscheidende Voraussetzung zur mechanistischen Erfassung 
des genetischen Einflusses und der Gen $x$ Umwelt-Beziehung ist. Unsere Ziele sind (i) molekulare und zelluläre Komponenten zu integrieren, um deren globale Funktion in einem relevanten synaptischen Netzwerk zu verstehen, (ii) die involvierten Hirnregionen und Verhaltenskorrelate zu erfassen, (iii) die Einflüsse der Umwelt auf diese Funktionen zu erkennen, und schlussendlich (iv) aus den neu gewonnenen Erkenntnissen Schlussfolgerungen für die klinische Praxis abzuleiten.

Diese Sonderausgabe von Neuroforum bietet einen Einblick in die einzelnen Forschungsbereiche. Carsten Wotjak und Hans-Christian Pape führen kursorisch in die synaptischen Schaltkreise von Furcht, Angst und Furchtextinktion ein, ehe Norbert Sachser und Klaus-Peter Lesch die Bedeutung von Gen $x$ Umwelt-Interaktionen am Beispiel von Tiermodellen gesteigerten Angst- und Furchtverhaltens erläutern. Anschließend dokumentieren Evelyn Glotzbach-Schoon, Marta Andreatta, Andreas Mühlberger und Paul Pauli, wie sich mithilfe virtueller Realität und in enger Anbindung an Tierstudien Modelle für pathologische Angst im Menschen entwickeln lassen. Katharina Domschke nimmt abschließend den Faden präklinischer Untersuchungen zu molekularen Grundlagen von Angst und Furcht auf, indem sie das Zusammenwirken von genetischen Faktoren und Umwelteinflüssen bei Angsterkrankungen des Menschen aufzeigt, ein neues Vulnerabilitätsgen vorstellt und dessen Bedeutung für Angsterkrankungen im Systemkontext nachweist.

In die wissenschaftlichen Arbeiten des SFB-TRR58 ist ein Nachwuchskonzept integriert, in das die beteiligten Standorte Hamburg, Münster und Würzburg ihre strukturierten Graduiertenprogramme mit relevanten Themenstellungen und methodischen Ansätzen einbringen. Umgekehrt stellen die Mitglieder des SFB-TRR58 ihre Expertise diesen Programmen zur Verfügung. Darüber hinaus bietet der SFB-TRR58 dem wissenschaftlich interessierten Nachwuchs auf früherer Stufe Möglichkeiten zur Erkundung wissenschaftlicher Arbeit. Dies geschieht beispielsweise im Rahmen von "Schülerlabors" direkt in den Forschungseinrichtungen oder durch Vorträge der SFBMitglieder in Leistungskursen der Biologie.

Insgesamt verknüpft der SFB-TRR58 die Forschung an drei Universitäten zum Thema Furcht/Angst/Angsterkrankungen, leistet einen substanziellen Beitrag zur
Profilbildung an den Standorten, generiert wichtige wissenschaftliche Beiträge im Forschungsfeld und leitet Ausbildungs- und Nachwuchsprogramme zur Neurobiologie der Emotion. Gleichzeitig zielt der SFBTRR58 auf die Heranführung einer ,neuen" Generation von Wissenschaftlerinnen und Wissenschaftlern an das Gebiet der Emotionsforschung und das vertiefte Verständnis der mechanistischen Prinzipien von Furcht und Angst. Aus diesen Ergebnissen erwarten wir langfristig eine Richtungsbestimmung zur signifikanten Verbesserung von Prognose, Diagnose und Therapie der Angsterkrankungen.

Dank gebührt der Deutschen Forschungsgemeinschaft für die Förderung unserer wissenschaftlichen Arbeit und allen Mitgliedern des SFB-TRR58, die dieses Verbundprojekt tragen.

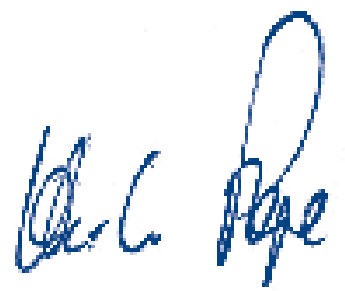

Prof. Dr. Hans-Christian Pape

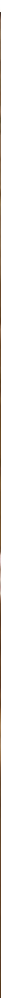

SHIPPING GLDBALL SINCE 1974

REQUEST A CATALDG AT FINESLIENLE.DE

口R CALL + 49 ( $\square) 6221-9 \square 5 \square 5 \square$

$5 \cdot 0$ ( 